\title{
Thermal conductivity and temperature profiles of the micro porous layers used for the polymer electrolyte membrane fuel cell
}

\author{
Odne S. Burheim, Huaneng Su, Sivakumar Pasupathi, Jon G. Pharoah and Bruno G. Pollet
}

\begin{abstract}
The thermal conductivity and the thickness change with pressure of several different micro porous layers (MPL) used for the polymer electrolyte membrane fuel cell (PEMFC) were measured. The MPL were made with different compositions of carbon and polytetrafluoroethylene (PTFE). A one-dimensional thermal PEMFC model was used to estimate the impact that the MPL has on the temperature profiles though the PEMFC.

The thermal conductivity was found to vary from as low as 0.05 up to as high as $0.12 \mathrm{~W} \mathrm{~K}^{-1} \mathrm{~m}^{-1}$ while the compaction pressure was varied from 4 bar and up to around 16 bar resulting in a decrease in thickness of approximately $40 \%$. The PTFE content, which varied between 10 and $25 \%$, did not result in any significant change in the compression or thermal conductivity. Both the thickness and the thermal conductivity changed irreversibly with compaction pressure.
\end{abstract}

Considering a MPL thermal conductivity of $0.1 \mathrm{~W} \mathrm{~K}^{-1} \mathrm{~m}^{-1}$, a MPL thickness of 45 $\mathrm{mm}$, a current density of $10 \mathrm{kA} \mathrm{m}^{-2}\left(1.0 \mathrm{~A} \mathrm{~cm}^{-2}\right)$, liquid water (production and sorption), and a $30 \mathrm{~mm}$ membrane it was found that the MPL is responsible for a temperature increase of up to $2 \mathrm{O}$. This contribution can be lowered by integrating the MPL into the porous transport layer.

\section{Introduction}

The low temperature Polymer Electrolyte Membrane Fuel Cell, PEMFC, converts the chemical free energy of the hydrogen oxygen reaction into electric work with high efficiency. For automotive applications, thermal management, degradation (ageing), and cost reduction are important factors for commercial success. The PEMFC comprises the Membrane Electrolyte Assembly (MEA) sandwiched between thin micro porous layers (MPL) and somewhat thicker porous transport layers (PTL). Until now, the thermal conductivity of the MPL has received little attention. In this paper we report the thermal conductivity of different MPL and discuss the impact of the MPL thermal conductivity on temperature control of the MEA. 


\subsection{Heat and work of the PEMFC}

The PEMFC is not only a very efficient energy converter, but also a very effective one. However, when increasing the current density (reaction rate), the reversible and irreversible heat release increases as well. This is for at least three reasons [1e7]. The temperature multiplied by the change in the reaction entropy and the current divided by the Faraday constant $(\mathrm{TDSj} / \mathrm{nF}$ ) is a reversible and inevitable heat source in a PEMFC. The Tafel (or ButlereVolmer) equation predicts that increased current densities necessarily result in additional losses, i.e. a reduction in the cell potential by an over-potential, $\mathrm{h}$, with a corresponding heat release. This is the origin of the second heat source which is numerically the product of the current and the overpotential (hj ) [8]. Finally, the Joule or ohmic heat is due to ohmic resistance (RUj ), most significantly in the membrane, and is given by $\mathrm{RUj}^{2}$.

According to Fourier's law of heat conduction, this heat release necessarily results in temperature gradients inside the PEMFC. Because almost all the heat sources are associated with the MEA, the thermal conductivity of the PTL and the MPL is more important than the thermal conductivity of the MEA itself. This is because these porous layers literally provide thermal insulation for the MEA. Whereas many of the mechanical properties and the thermal conductivity of the PTL is now starting to be understood and thoroughly investigated, the MPL thermal conductivity is not as well addressed [9e11]. What is known, is that for some combinations of MPL and PTL the average or combined effective thermal conductivity is not significantly different $[12,13]$. One reason why the pure MPL thermal conductivity is overlooked can be related to the fact that the MPL is one order of magnitude thinner than the PTL. However, in this paper we demonstrate that the thermal conductivity is also one order of magnitude larger for the MPL compared to some types of PTL. Because of this relation, the thermal conductivity of these two materials are equally important.

\subsection{The role of the MPL in a PEMFC}

Often a MPL is included in the MEA structure, between each backing layer (i.e. PTL) and catalyst layer (CL), as illustrated in Fig. 1. The MPL consists of carbon black powder and a hydrophobic agent, usually polytetrafluoroethylene (PTFE). The function of the MPL is to provide proper pore structure and hydrophobicity to allow a better gas transport and water removal from the CLs, and also to minimise electric contact resistance between the CL and the macroporous PTL substrate (usually carbon paper or carbon cloth) by forming a flat and uniform layer [14].

With respect to the importance of achieving high performance of PEMFC, extensive work has been performed to examine how the MPL properties such as (i) carbon powder type, (ii) carbon loading (or thickness), and (iii) PTFE content 
control the water management in PEMFC [15e21]. In contrast to that, the literature about how the MPL properties affect the heat management in the PEMFC is limited $[13,22,23]$, although this is also very important for real fuel cell applications. The reason for this being important, is that the water saturation pressure increase significantly with temperature such that small temperature variations in the range from $75^{\text {to }} 95^{\circ} \mathrm{C}$ can significantly change the local performance and degradation of a PEMFC.

\subsection{Thermal conductivity measurements of PEMFC components}

Good measurements of the thermal conductivity of PEMFC materials are challenging for many reasons. For the PTL, the in-plane thermal conductivity is different from the throughplane thermal conductivity. This was first postulated [24e26] because the structure of the materials and the electric conductivity are not isotropic. Recently this was also experimentally shown [27e29] and the in-plane value was found to be five to ten times larger than the through-plane value (depending on the PTL compaction). When measuring the through-plane thermal conductivity, the thermal contact resistance, the thermal conductivity and the thickness change with the applied compaction pressure must be accounted for [30e32]. Separating the thermal contact resistances from the material thermal resistance is a challenge associated with this. Both water and PTFE change the thermal conductivity of the PTL [31e34]. In this section we give a brief review of measurements and modelling of the thermal conductivity of PEMFC materials.

Mathematical models have also been applied as an important supporting tool to supplement the thermal conductivity measurements of PTL materials. By combining calculation and measurements, Ramousse et al. [30] estimated minimum and maximum thermal conductivities of carbon papers based on a previously developed model [25], connecting the thermal resistances of the solid and gas phases in parallel and in series.

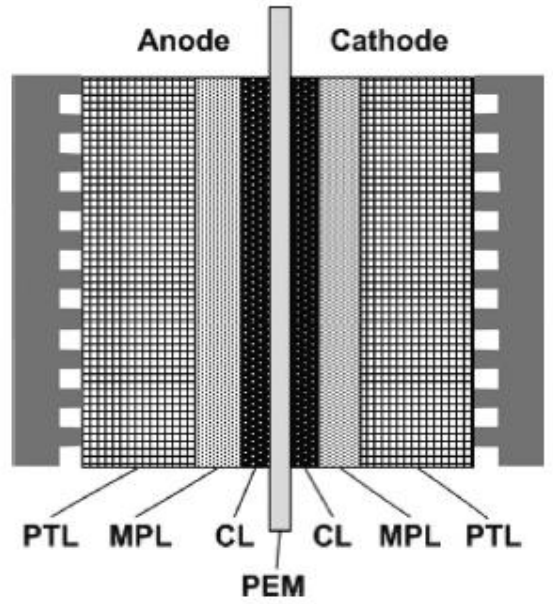

Fig. 1 - Schematic diagram of a 7-layer structure MEA with MPL sandwiched between two PTL in turn between two polarisation plates. 
Sadeghi et al. [26] reported an analytical model to predict thermal conductivity based on the material structure, simplified by assuming a basic repeating structure. Recently, Yablecki et al. reported a model of thermal conductivity including PTFE under various loads [35].

Together with precise thickness measurements, determining the thermal contact resistances between the apparatus and the samples and also between the samples when stacked are the most important elements when conducting thermal conductivity measurements [30]. Ramousse et al. stated that the contact resistance should be bound by the resistance of layers of either air or carbon of the thickness of an individual PTL fibre. Previously we reported the contact resistance to be approximately $2 \times 10^{-4} \mathrm{~m}^{2} \mathrm{~K} \mathrm{~W}^{-1}$ [31] which, if considering a fibre thickness of about $5 \mathrm{~mm}$, refers to a thermal conductivity of the contact region close to $0.025 \mathrm{~W} \mathrm{~K}^{-1} \mathrm{~m}^{-1}$. This value is the same as for air. When stacking any samples in the measurement, a new contact resistance emerges e namely the sampleesample contact resistance. The challenge associated with this resistance is that for every added sample one also adds one more sampleesample contact resistance. This procedure is often done to change the thickness of the sample so that one can find the ratio between the thickness and the thermal resistance of the material or the thermal conductivity. However, since one also increases the sampleesample contact resistance there are more unknowns than equations. In several measurements we have shown that the sampleesample thermal contact resistance is in fact negligible [12,31]. Regardless of this, we include an appendix, A, to justify which thermal contact resistances are significant.

The first in-situ measurement for the through-plane thermal conductivity was reported by Vie et al. [36]. Embedding thermo-couples in the gas channels and between the membrane and the catalyst layer and accounting for the known heat release, a thermal conductivity of $0.19 \pm 0.05 \mathrm{~W} \mathrm{~K}^{-1} \mathrm{~m}^{-1}$ for an ETEK ELAT PTL was estimated. The first ex-situ experiments of thermal resistance (of the sample and the contact to the apparatus) were reported by Ihonen et al. [37]. Khandelwal and Mench [34] reported the first ex-situ measurements of PTL materials where the thermal conductivity and the thermal contact resistance to the apparatus was deconvoluted. In this study, the compression, and thus the actual thickness, was not measured - lowering the precision of the reported values. Ramousse et al. [4] used a similar approach. Their apparatus applied copper plates at the end of each cylinder, sandwiching the investigated sample. Using this experimental set-up, different PTL materials provided by Toray (3 different thicknesses) and SIGRACET were tested by measuring the thermal resistance of stacks of samples. The first report on ex-situ measurements accounting all of the three parameters required by Fourier's law and as a function of compaction pressures was that of Burheim et al. 
[31]. When correcting for the actual thickness due to the compression, the reported thermal conductivity values change by $5 \mathrm{e} 20 \%$. Perhaps the most important part of this study was that we demonstrated that the PTLePTL contact thermal resistance was negligible such that stacks of samples could legitimately be used to vary the sample thickness and to separate the through-plane thermal conductivity from the contact resistance with the apparatus.

When the temperature changes, the material properties can also change. The change in thermal conductivity with temperature of PTL (both in- and throughplane) was measured by Zamel et al. [29,38]. For the through-plane thermal conductivity with thickness controlled compression; it was found that at $16 \%$ compression (unknown compaction pressure) the thermal conductivity of the PTL, regardless of PTFE content, does not depend significantly on temperature [38]. For the in-plane thermal conductivity it was found that for PTFE free PTLs the thermal conductivity is lowered by $\mathrm{w}_{50} \%$ when comparing values measured at room temperature to values from measurements undertaken at $60{ }^{\circ} \mathrm{C}$ and higher [29]. For the PTFE treated samples, the in-plane thermal conductivity is nearly unaffected in the range of -20 to $\mathrm{p} 120 \mathrm{O}$, respectively [29]. This is similar to what Khandelwal reported for Nafion [34].

The most recent contributions to the knowledge of thermal conductivity of the PTL is the effect of ageing [12] and the role of the so called heat pipe effect in PTLs [33]. Ageing removes PTFE in the PTL. The PTFE appears to be removed only at the locations away from fibre-to-fibre contact such that the thermal conductivity of the dry PTL remains unaffected. However, the ageing makes the PTLs more susceptible to water and therefore the thermal conductivity of aged wet PTLs is higher than that of pristine wet PTLs [12]. Another subject that is recently reported on is the heatpipe-effect. This is the effect of heat transported by vaporisation and condensation in a porous (or a pipe) in a thermal gradient. When PTLs are wet and at temperatures above $70 \mathrm{O} \mathrm{C}$ the thermal conductivity of the PTL is increased by $20 \mathrm{e} 40 \%$ [33].

In many of the first studies, the thermal conductivity of the PTL, the MPL coated PTL and the membrane (Nafion) was reported [31,34]. Recently, Unsworth et al. reported a study on SolviCore PTLs with and without MPL [13]. The study shows that adding the MPL to the PTL only slightly lowered the effective thermal conductivity of the layer and only at higher compaction. Later, for Sigracet carbon papers, it was found that the MPL coating does not significantly change the overall effective thermal conductivity [12]. The thermal conductivity of the MPL in itself without the supporting PTL is still not (to our knowledge) studied and reported. For the first time we study and report the thermal conductivity and compression of the MPL in the absence of the PTL substrate. We are also for the first time, to our knowledge, discussing how the temperature profile is affected by the MPL properties. 


\section{Theory and experimental}

\subsection{Apparatus}

The apparatus used in the experiments is the same as the one reported in previous publications $[12,31,32]$ and is depicted in Fig. 2. In brief, the heat passing through a sample along with the temperature difference across the sample is measured. This gives us the thermal resistance of the investigated sample, RSample. The sample can be a stack of materials or a single layer. Here, the sum of the sample- and the contact thermal resistance, $R$ Sample $\mathrm{p} 2 R$ App.-Sample is measured.

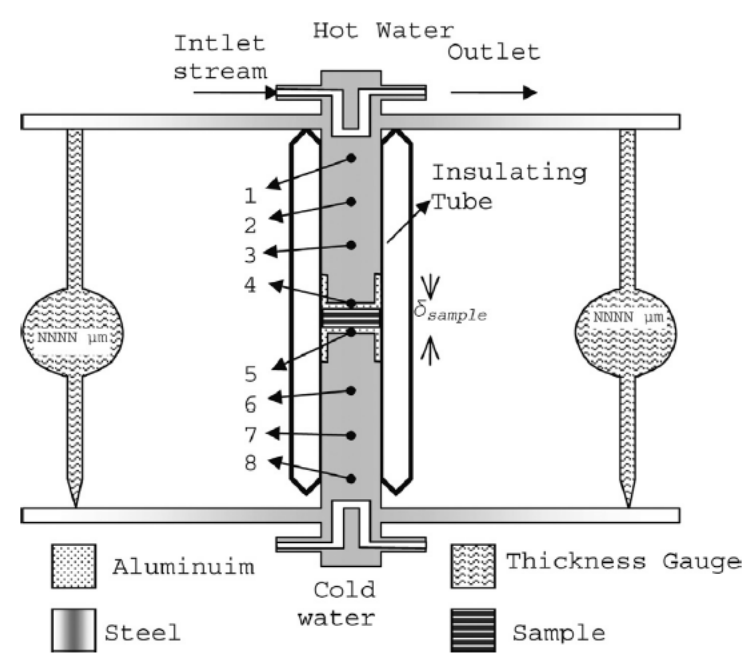

Fig. 2 - A 2D sketch of the apparatus used to measure thermal conductivity as reported here $[31,32]$.

\section{Table 1 - The PTFE content of the investigated MPLs.}

\begin{tabular}{lc} 
Sample & PTFE/\% \\
\hline MPL10 & 10 \\
MPL15 & 15 \\
MPL20 & 20 \\
MPL25 & 25 \\
\hline
\end{tabular}

In more detail, a heat flux is set up in two cylindrical pistons (sandwiching the investigated sample) by flowing temperature controlled water in the upper and lower part of the apparatus. This heat flux is measured in each part of the apparatus by three thermocouples, i.e. $1 \mathrm{e} 3$ and $6 \mathrm{e} 8$. By calibration one can determine the thermal conductivity of the stainless steel and thus, by Eq. (1), obtain the heat flux through the investigated sample. At the end of the two pistons, in contact with the investigated sample, a small cap of a highly conductive material is placed. This material contains thermocouples (4 and 5) and are screwed onto the pistons. Because of the high thermal conductivity, these caps are nearly isothermal and thus they act as enlarged thermocouples adjacent to the investigated sample. Thus one can measure the temperature drop across the investigated sample and its contact to the piston and, by Eq. (2), the sample and apparatus double contact 
resistance. By using calibrated micrometer gauges, the actual thickness was measured.

$$
\begin{aligned}
& q_{\text {upper }}=k_{\text {steel }} \frac{\mathrm{T}_{1}-\mathrm{T}_{3}}{\delta_{1-3}} \text { and } q_{\text {lower }}=k_{\text {steel }} \frac{\mathrm{T}_{6}-\mathrm{T}_{8}}{\delta_{6-8}} \\
& q_{\text {Sample }}=\frac{q_{\text {upper }}+q_{\text {lower }}}{2} \text {, and; } R_{\text {Sample }}+2 R_{\text {App.-Sample }}=\frac{T_{4}-T_{5}}{q_{\text {Sample }}}
\end{aligned}
$$

\subsection{The micro porous layers}

In total four different MPLs were prepared. These were made by spraying several layers of carbon particles, PTFE and a solvent on a thin copper film $(28 \pm 2 \mathrm{~mm})$. Subsequently the layers where heated to remove the solvent. The PTFE contents are given in Table 1. In order to prepare carbon ink for the MPL, carbon powder (Vulcan XC72, Cabot, USA) was mixed with PTFE-dispersed water (6o wt\% PTFE, Alfa Aesar) and isopropyl alcohol in an ultrasonic bath for $1 \mathrm{~h}$. The resulting carbon ink was spray-deposited onto one side of the copper foil (annealed, 99.8\%, Alfa Aesar), followed by drying at $80 \mathrm{o} \mathrm{C}\left(\mathrm{N}_{2}\right.$ atmosphere) for $2 \mathrm{~h}$ to evaporate all remaining solvent, and then at $350{ }^{\circ} \mathrm{C}\left(\mathrm{N}_{2}\right.$ atmosphere) for 30 min to uniformly distribute PTFE throughout the MPL [39]. The thickness of the layers were controlled by adding several MPL layers on top of each other.

\subsection{Procedure}

For this research the investigated material was stacked. This was done by taking a disk of the prepared MPL on copper along with a disk of pure aluminium (99.9\%) on top of the MPL. Thus the MPL is sandwiched between a thin disk of copper and one of aluminium. This unit was investigated either as single or in a stack of two units (in order to vary the thickness as much as possible).

Looking at Eqs. (1) and (2), one can see that knowledge of the sample thickness is important. The metal films sandwiching the MPL sample were chosen especially because of their high thermal conductivity. Thus these films will not contribute directly to the measured thermal resistance, $R$ App.- Sample $\mathrm{b} 2 R$ App.-Sample. However these materials will contribute to the measured thickness of the sample. Therefore the thickness of these films was measured and subtracted for before plotting and calculating the thermal conductivities.

\subsection{Statisticalanalysis and accuracy of the measurements}

The thermal conductivity apparatus was calibrated using materials with known thermal conductivity, see Ref. [31]. These values are known with $5 \%$ accuracy and thus this is the accuracy limitation of the reported values in this paper [31]. The

\section{http://repository.uwc.ac.za}


thermal conductivities in the result section reports deviations from the linear regression using a least square of residual approach. All numbers are reported with 95\% confidence intervals. When subtracting for the aluminium and copper film thicknesses the variance, $s i^{2}$, will propagate as given by the $\mathrm{r}$ of propagations formula, Eq. (3).

$$
\sigma_{i}^{2}=\sum_{n=1}^{i}\left(\frac{\partial f\left(x_{1}, x_{2}, \ldots, x_{i}\right)}{\partial x_{n}} \sigma_{n}\right)^{2}
$$

\subsection{Temperature distribution model}

Mathematical models of fuel cells accounting for temperature gradients have become more common over the last decade, as are well summarised by Bapat and Thynell [40] and by Zhang and Khandlikar [41].
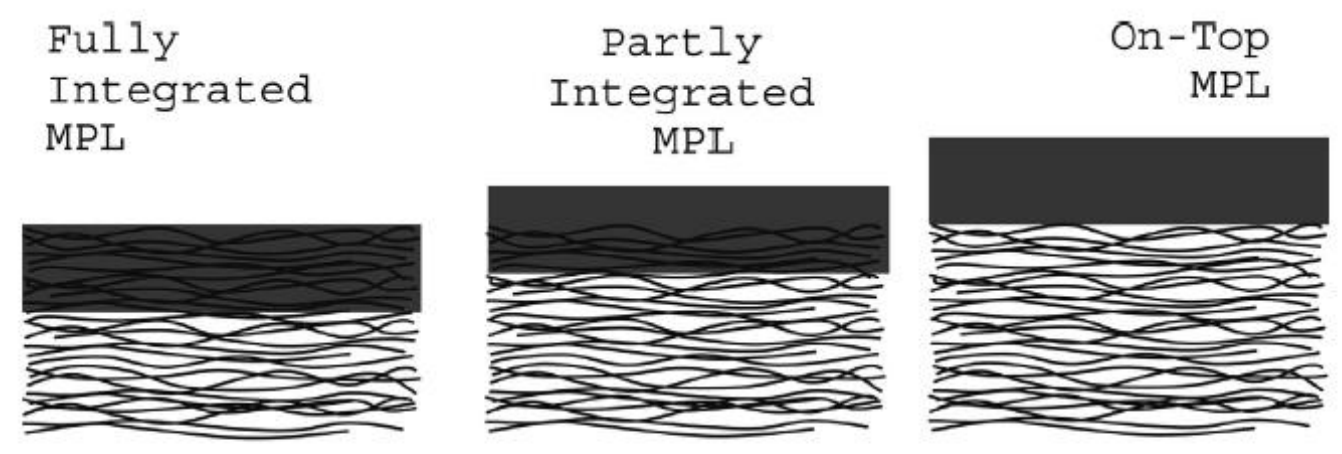

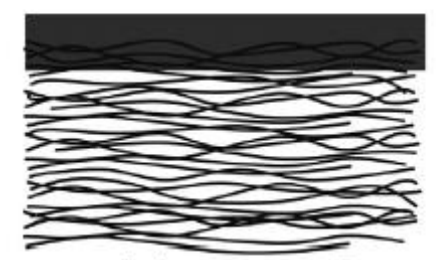

Thin Partly

Integrated MPL

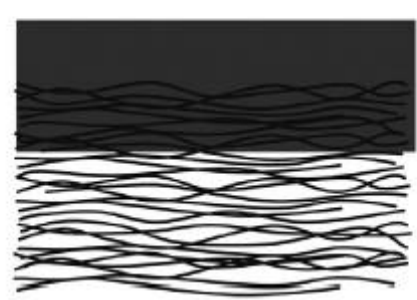

Thick Partly Inteqrated MPL

Fig. 3 - Graphical description of the different MPL-PTL integration considered in the temperature model.

Highly complex and dynamic models can be developed. In this study, however, we are only interested in evaluating the impact that the MPL and that MPLePTL integration has on the temperature profiles through the PEMFC. In Fig. 3, several illustrations of potential MPL-PTL integrations are given. Because the MPL is typically deposited on top of the highly porous PTL (typically around 80\%), it will to some extent be integrated with the PTL. Therefore, the "On-Top MPL" in Fig. 3 is not considered very likely and is not considered in this study. The four other scenarios are considered. If the MPL is fully integrated in the PTL, there will be no 
layer with a thermal conductivity equivalent to the values reported in this paper. This is because from previous studies $[12,13]$ it is known that the effective thermal conductivity is not affected by the MPL when fully integrated in the PTL, at least in a dry state. Thus this MPLePTL configuration was chosen as a base case and with the MPL-only thickness equal to zero, see Table 2. Furthermore, three configurations were considered where there is a MPL on the top of the PTL. In Fig. 3, these can be seen as thin, normal and thick MPL partly integrated in the PTL. These configurations, thin, normal, and thick partly integrated MPL, are represented by 15 , 30, $45 \mathrm{~mm}$ thick pure MPL on top of the PTL in the model (see Table 2), correspondingly. In order to justify the actual choice of MPL thickness's in our model, a SEM micrograph of a freeze-cut of the core of a PEMFC was studied, see Fig. 4. In this figure, the PEMFC consisted of two commercially MPL coated PTL (Freudenberg FFCT H2315 3X196) sandwiching a custom made MEA (0.4 $\mathrm{mg} \mathrm{Pt} \mathrm{cm}^{-2}$ on a Nafion 212). The MEA in the SEM micrograph was prepared by using the catalyst coated membrane (CCM) method. (Catalyst inks were prepared by dispersing catalyst powder (Hispec $4100 \mathrm{Pt} / \mathrm{C}$, Johnson Matthey) into a mixture of isopropanol and $5 \mathrm{wt} \%$ Nafion ionomer solution (DuPont, USA). The dispersion mixture was ultrasonicated for 30 min before being used. Then, the inks were sprayed on both sides of Nafion 212 membrane (DuPont, USA) to form the catalyst layers.) The freeze cut was made by immersing this PEMFC in liquid nitrogen and then cutting with a scalpel. This figure is shown here to demonstrate the degree of realism in the MPLePTL configuration discussion above. The figure also shows the region chosen to represent the model and how this model is geometrically linked to a PEMFC.

Moreover, the PTL in the region was considered to contain some residual water, to be operated with backing plates at $70^{\circ} \mathrm{C}$, and with a current density of 10 ooo $\mathrm{A}$ $\mathrm{m}^{-2}\left(1 \mathrm{~A} \mathrm{~cm}^{-2}\right)$. A cathode over potential of $\left(0.447 \mathrm{p} 0.058 \ln \left[\mathrm{j} / 10^{4}\right]\right)^{1}[2]$ was assumed and a conductivity of $8.7 \mathrm{~S} \mathrm{~m}^{-1}$ [42] and a Nafion 111 membrane, this current density corresponds to a cell potential of approximately $0.7 \mathrm{~V}$. The reaction is considered to produce liquid water and not to adsorb any water from the gas vapour phase. Thus, the cathode reaction entropy is close to $163 \mathrm{~J} \mathrm{~mol}^{-1} 2 \mathrm{~K}^{-}$ 1 ; because most of [5] or large portions [7] of the reaction entropy for a PEMFC in operation is measured to be associated with the cathode and because liquid water absorbing and desorbing from a well humidified Nafion membrane is close to zero [43]. Regarding the cathode heat sources, F is the Faraday constant, DS the reaction entropy, and $\mathrm{j}$ the current density as $\mathrm{A} \mathrm{m}^{-2}$. It was considered that the PTL was a slightly wetted Toray TGP-090 10\% wet proof with a thermal conductivity twice that of the dry value at w14 bar compaction pressure, i.e. $2 \times 0.48 \mathrm{~W} \mathrm{~K}^{-1} \mathrm{~m}^{-}$ 1 [32]. The MPL layer that is on top of the PTL is considered to have a thermal conductivity of $0.10 \mathrm{~W} \mathrm{~K} \mathrm{~K}^{-1} \mathrm{~m}^{-1}$, according to the measurements reported in this paper. The electrode layer is considered to have a similar thermal conductivity as the

\section{http://repository.uwc.ac.za}


MPL, however rather thin. The membrane is considered a well humidified Nafion 112 with a thermal conductivity of $0.25 \mathrm{~W} \mathrm{~K}^{-1} \mathrm{~m}^{-1}$ [31].

Table 2 - The model parameters used for the model in this paper.

\begin{tabular}{lccc} 
Material & $\mathrm{k} / \mathrm{W} \mathrm{K}^{-1} \mathrm{~m}^{-1}$ & $\delta_{i} / \mu \mathrm{m}$ & $\widehat{\mathrm{Q}} \cdot \delta_{\mathrm{i}} / \mathrm{W} \mathrm{\textrm {m } ^ { - 2 }}$ \\
\hline Backing & 200 & $\geq 100$ & - \\
Contact & 0.7 & 5 & - \\
PTL & 0.96 & 255 & - \\
MPL & 0.10 & $0,15,30,45$ & $0.001 j$ \\
Anode & 0.1 & 10 & $\left(0.447+0.058 \ln \frac{j}{10^{4}}+\frac{\mathrm{T} \Delta S}{2 \mathrm{~F}}\right) \mathrm{jj}$ \\
Cathode & 0.1 & 20 & $\left(\delta_{i} / 8.7\right) j^{2}$
\end{tabular}

The anode and the cathode backing plates are considered nearly isothermal, and to have a thermal conductivity similar to aluminium, i.e. $200 \mathrm{~W} \mathrm{~K}^{-1} \mathrm{~m}^{-1}$ [44]. The outside temperature is set to $70^{\circ} \mathrm{C}$ (Dirichlet boundary condition.). The thermal contact between the backing and the PTL can be very large for dry materials, but here a value according to a wetted interface, i.e. $0.710^{-4} \mathrm{~K} \mathrm{~m}^{2} \mathrm{~W}^{-1}$ [32], was considered. In the model, this is implemented by adding a region of $5 \mathrm{~mm}$ with a thermal conductivity of $0.7 \mathrm{~W} \mathrm{~K}^{-1} \mathrm{~m}^{-1}$. All the model properties discussed in this section are listed in Table 2.

The model is developed in the commercial software Comsol 4.2a which is set to solve

$$
\nabla\left(k_{i} \nabla \mathrm{T}\right)+\widehat{Q}_{\mathrm{i}}=0
$$

where $k i$ is the thermal conductivity and etric heat source for region $i$ as given in Table 2 . The model was solved for quadratic mesh and checked for mesh independence by doubling the mesh resolution.

\section{Results and discussion}

We report first the thermal conductivity of the MPL, Section 3.1, next the effect of compression and compression hysteresis, Section 3.2 and finally possible implications of PEMFC cross sectional temperature profiles, Section 3.3. The method validation is given in detail in Appendix A.

\subsection{Thermal conductivity of the MPL}

The measured thermal resistance of the MPL containing 25\% PTFE at 4.6 and 16.1 bar compaction pressure are shown in Fig. 5. The inverse of these slopes gives the thermal conductivity of the MPL at the given pressure. One can see that the measured thermal resistance is highly linear with the thickness. This is also 
reflected in the thermal conductivities obtained from the regression analysis, which at these two pressures were determined to be $0.073 \pm 0.007$ and $0.087 \pm$ $0.012 \mathrm{~W} \mathrm{~K}^{-1} \mathrm{~m}^{-1}$ at 4.6 and $16.1 \mathrm{bar}$, respectively. This procedure was repeated for the MPL containing 10, 15, 20, and 25\% PTFE. This was done at different compaction pressures - first while increasing the pressure stepwise from 4.6 to 16.1 bar and then, finally, by reducing the pressure in one step back to 4.6 bar. All these results are tabulated in Table 3 and the values obtained under increasing and decreasing compaction pressure is indicated by superscript $\backslash$ and $\mathrm{Z}$ respectively. The thermal conductivity values that were obtained while increasing the compaction pressure are shown graphically as a function both of composition and compaction pressure in Fig. 6. From this figure it can easily be seen that it is compaction pressure that is the principal component when the thermal conductivity changes. To emphasise this even further, the thermal conductivity of the different MPL compositions are shown as function of the applied compaction pressure in Fig. 7. One can clearly see that it is the compaction pressure rather than the PTFE content that dictates the change on the thermal conductivity.

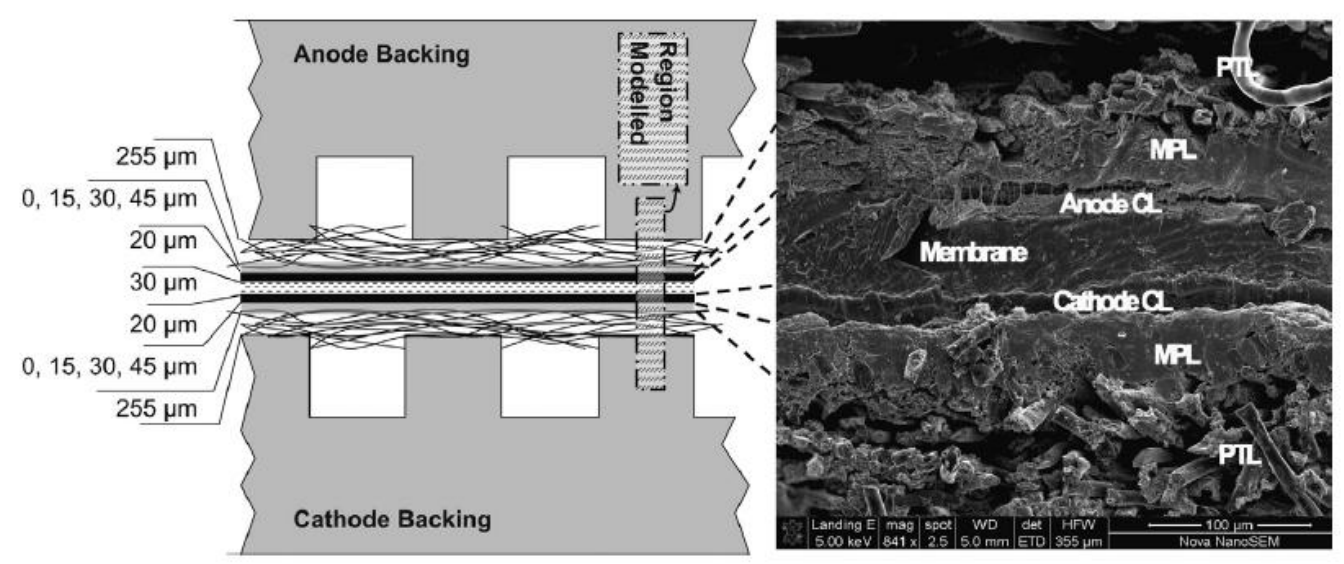

Fig. 4 - The model geometry (left) used in this paper and a SEM micrograph (right) illustrating the link to the chosen geometric parameters.
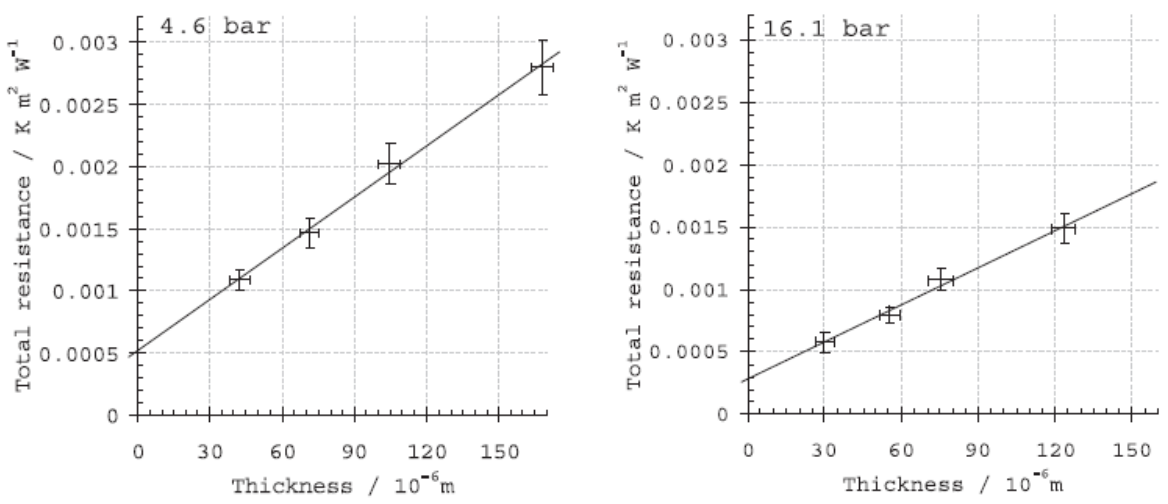

Fig. 5 - The total measured thermal resistance for the MPL containing 25\% PTFE at 4.6 and 16.1 bar compaction pressure. 


\subsection{Compressibility of the MPL}

The thermal conductivity cycle of all the materials and the thickness cycle (of a sample containing 20\% PTFE) as functions of compaction pressure can be seen in Fig. 8. From this figure, one can see how strong the relation between the compression and the thermal conductivity is. One can also see that the compression is almost completely irreversible. This means that when the MPL is compressed in a PEMFC, it is the highest compaction applied that will dictate the thermal conductivity of the layer.

Another aspect that should also be clear is that the thermal conductivity of this soft material relies on contact between the material particles within. This study does not consider the gas phase tortuousity of the MPL under compression. As the material is compressed this property is likely to increase, and the increased thermal conductivity might come at the cost of reactant and product gas transport properties.

As was discussed in the introduction [45], some fibrous carbon papers used as PTL exhibit partial hysteresis in thickness when exposed to cyclic compression. When these materials are compressed, some of the fibres snap or break while others remain intact. Because some fibres break and some do not, the PTL is not entirely elastic and not fully irreversibly compressed. Because the PTL is the support for the MPL, the hysteresis of compression for the MPL and the PTL should be seen together. For instance, when the membrane expands and contracts when undergoing hydration cycles (e.g. during start-up and shut-down of the PEMFC systems); The MPL can be expected to keep a constant thickness due to the first compressions whereas the PTL will contract and expand elastically. Hence the PTL thermal conductivity can be expected to be dynamic and the MPL thermal conductivity static. 


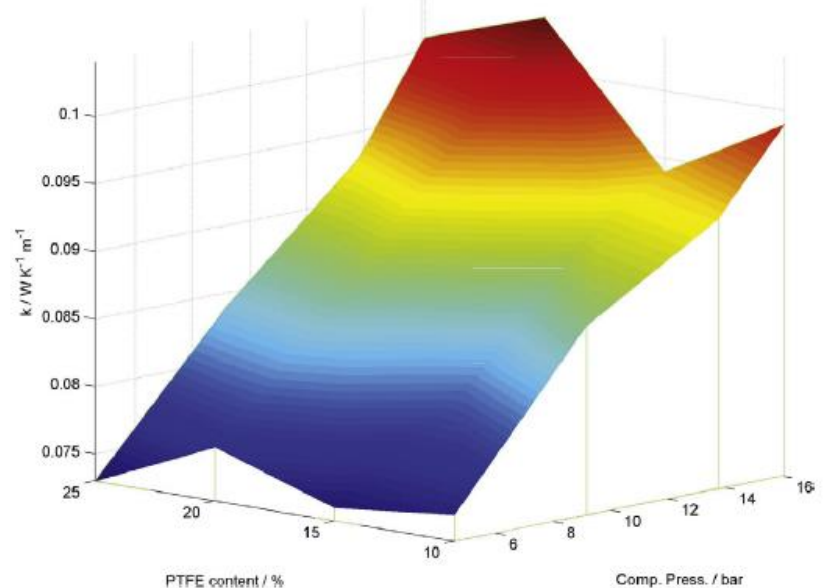

Fig. 6 - The measured thermal conductivity of the MPL as a function of applied compaction pressure and PTFE content.

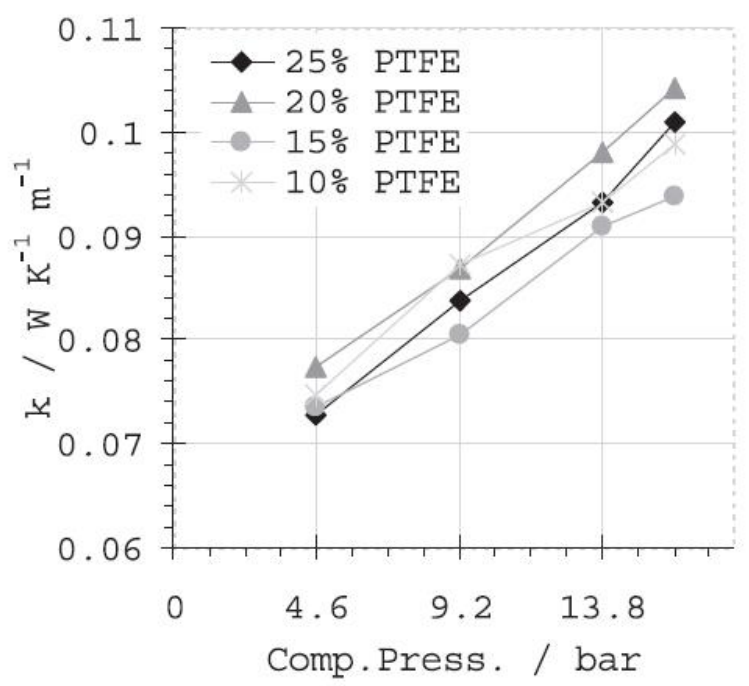

Fig. 7 - The measured thermal conductivity of the MPL as a function of applied compaction pressure for different PTFE content.

Table 3 - Overview of measured thermal conductivities for various MPLs at different compaction pressure. The thermal conductivities are given as $\mathrm{mW} \mathrm{K}^{-1} \mathrm{~m}^{-1}$ and the lowest row shows the thermal conductivity at 4.6 bar compression after first being compressed at 16.1 bar.

\begin{tabular}{lccrr} 
P/bar & $10 \%$ PTFE & $15 \%$ PTFE & $20 \%$ PTFE & $25 \%$ PTFE \\
\hline $4.6^{\Uparrow}$ & $75 \pm 13$ & $74 \pm 20$ & $77 \pm 11$ & $73 \pm 7$ \\
$9.2^{\Uparrow}$ & $87 \pm 15$ & $81 \pm 22$ & $87 \pm 18$ & $84 \pm 11$ \\
$13.8^{\Uparrow}$ & $93 \pm 13$ & $91 \pm 19$ & $98 \pm 21$ & $93 \pm 13$ \\
$16.1^{\Uparrow}$ & $99 \pm 19$ & $94 \pm 26$ & $104 \pm 21$ & $101 \pm 13$ \\
$4.6^{\Downarrow}$ & $92 \pm 6$ & $84 \pm 8$ & $88 \pm 20$ & $87 \pm 12$ \\
\hline
\end{tabular}



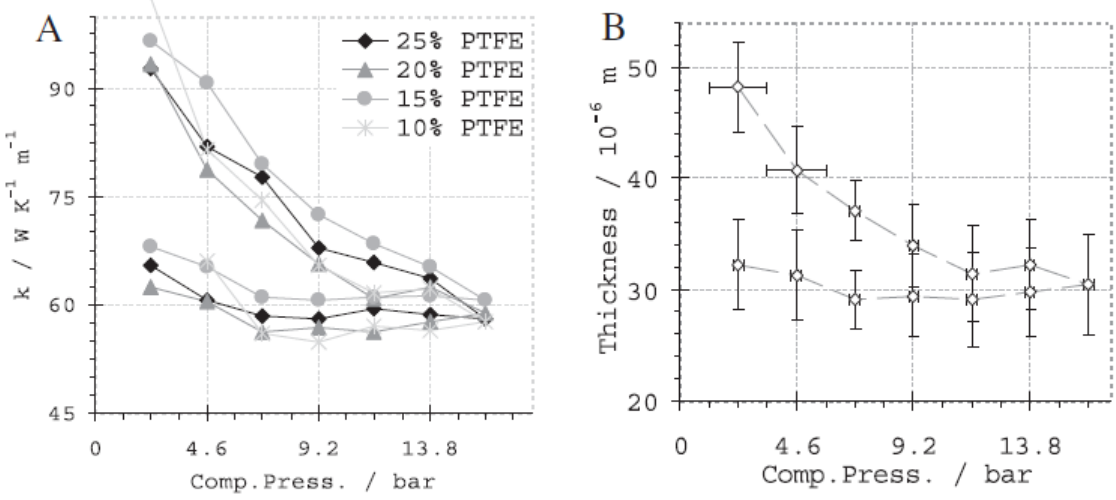

Fig. 8 - All the thermal conductivity values (A) and a single sample (20\% PTFE) thickness (B) as function of the compaction pressures during a compaction cycle going up to 16.1 bar and down again.

\subsection{MPL impact on the PEMFC internal temperature profiles}

As described in Section 2.5, this study includes a simple one dimensional model in order to illustrate the impact of the reported MPL thermal conductivity. The modelled temperature profiles are shown in Fig. 9. The only change between the four different temperature profiles is the assumed thickness of the MPL (on top of the PTL). This layer is to the left of the anode and to the right of the cathode. One can see that despite the relatively thin MPL, it can cause an almost doubling of the temperature difference between the warmest and the coldest point inside the fuel cell. At a current density of $10000 \mathrm{~A} \mathrm{~m}^{-2}\left(1.0 \mathrm{~A} \mathrm{~cm}^{-2}\right)$ the temperature increase due to the MPL is between $\mathrm{O}$ and $2{ }^{\circ} \mathrm{C}$. This corresponds to a thermal gradient of $36000 \mathrm{O} \mathrm{C} \mathrm{m}^{-1}$, which is enormous. It is the very low thermal conductivity in combination with the large heat generation in the effective MEA that is the reason for this.

The model consider the largest reported MPL thermal conductivity and in a region of a PEMFC which is usually partly flooded with water [46]. This means that the relative increase in temperature due to the MPL (almost 100\%) is the largest possible. If one considers the MPL to be dry, one can expect a temperature jump between the backing and the PTL of around $1{ }^{\circ} \mathrm{C}$ and that the temperature gradient in the PTL to double. In this dry case, the PEMFC total temperature difference contribution from the MPL goes from a maximum relative contribution of $50 \%$ to a minimum of $20 \%$.

In the end of this section, we would like to point out that by fully integrating the MPL into the PTL one can partly or fully lower the PEMFC internal temperature increase contribution from the MPL. If the PTL is dry, the thermal conductivity of the PTL is not affected by the integrated MPL [12]. Thus we have three ranges of thermal conductivity, high (wet PTL), intermediate (dry PTL or MPLePTL), and low (MPL external layer). When implementing the MPL into the PTL, around a tenth of the PTL 
thickness is replaced with a region with thermal conductivity of the dry PTL. When water is absent, the thermal conductivity of this integrated layer remains constant. When water is present, around ten percent of the PTL induced temperature drop is replaced with a larger temperature drop from the MPLePTL region. When water is absent, integrating the MPL does not affect the original PTL temperature drop. Thus it is under dry conditions (in comparison to the wet conditions) that the temperature drop is lowered the most by integrating the MPL into the PTL.

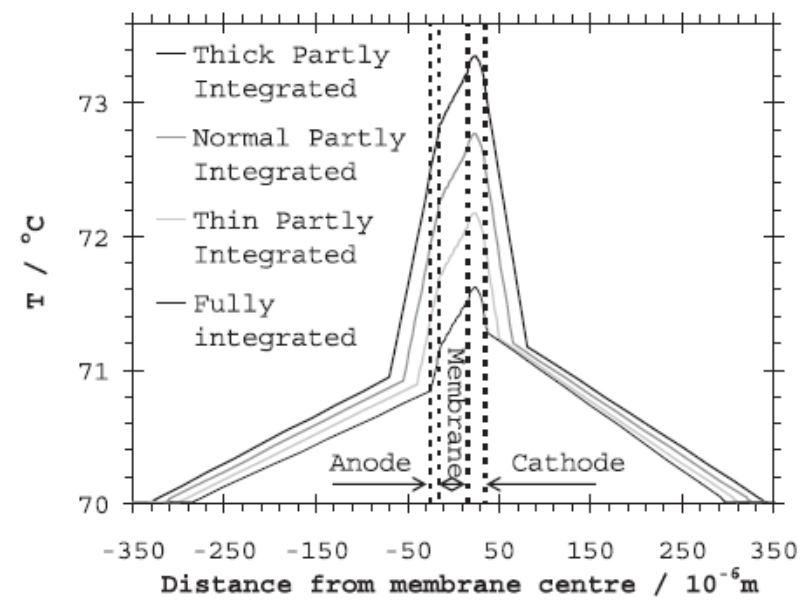

Fig. 9 - Modelled temperature profiles for different thickness of MPL not integrated in the PTL, according to Fig. 3.

\section{Conclusion}

Thermal conductivity and the compressibility of micro porous layers (MPL) with different PTFE content made for the PEMFC is measured and reported. The thermal conductivity varied from as low 0.05 to as high as $0.12 \mathrm{~W} \mathrm{~K}^{-1} \mathrm{~m}^{-1}$ when the compaction pressure changed from approximately $4 \mathrm{e} 16$ bar. The measured drop in thickness with compression was as high as 40\%. Both the thickness and the thermal conductivity changed irreversibly with compaction. Using a thermal model it was found that for $10000 \mathrm{~A} \mathrm{~m}^{-2}\left(1.0 \mathrm{~A} \mathrm{~cm}^{-2}\right), 30 \mathrm{~mm}$ membrane, and when only liquid water was considered, a $45 \mu \mathrm{m}$ thick MPL can increase the maximum internal temperature by $2{ }^{\circ} \mathrm{C}$.

\section{Acknowledgement}

The Norwegian Research Council is acknowledged for financial support, FRIENERGI, grant number 197598 and the RENERGI, grant number 164466/S30.

\section{Appendix A. Validation of procedure.}

In the present work one main approach of determining the thermal conductivity of the custom made MPL was chosen. This approach is based on measuring the thermal resistance of a single or a stack of two discs of MPL on a copper substrate with an additional layer of pure aluminium on top. Within total four such discs per material for investigation, the study was left 
with a minimum of the requirement for a linear regression and a corresponding variance. The chosen method allows for at least three critical questions against the choice of this procedure.

One argument against the chosen approach is that three points are too little to give a reasonable estimate on the thermal conductivity value and its variance because one should use at least five points to have a valid regression and variance. This argument is wrong. The fact is that one can severely lower the variance by increasing the number of measurements and that when one has five measured values the equations giving the variance is lowered enough (because of the denominator (n-2)) to be in a state where systematic errors (calibration of measurements) becomes more important than the random scatter of the measurements. What is also important in a least square residual approach is the distance between the points in the regression. For instance, in the present case it is better to measure three single points with a large thickness range than to measure many single points with a low thickness range. In the reported measurements it was strived to increase the range while keeping the number reasonably large (four).

A second argument against the chosen approach is the loss of precision in the thickness measurement when subtracting the $\mathrm{Cu}$ - and $\mathrm{Al}$-film thicknesses, and in particular when considering the "single" thickness MPL. The error of the thickness measurements when subtracting for these thicknesses propagates and decreases the precision of the measurements. However, also on thicker MPL were measured and it was taken care to make sure that every layer is compressed in a reproducible manner. This suggests that it is indeed better to have a reproducible layer of aluminium on top of each disc of MPL than to have different contacts for the single and the stacked materials.

The third argument against the chosen approach should be introduction of several contact resistances and because of the linear coupling between contact resistances and samples one is left with with a matrix without a determinant. This argument is only valid if the contact resistances of "MPL-Aluminium" and "aluminium-heated copper" are as large or larger than the order of a tenth of the resistance of the (double) MPL. This topic was debated extensively by Ramousse et al. [30]. There are two ways that one can use to test the lack of significance of these contact resistances.

One approach is to test the significance of the stack internal contact resistances is to change the thickness of the MPL. Using MPL with 1, 2, and 3 gcarbon $\mathrm{cm}^{-2}$, validation section) the measured total resistances for single and paired MPL (where only the thickness is changing) were plotted. This is shown in Fig. A.10. Because all the measured data points fit with the linear regression line (dashed) it was concluded that these contacts are of no relevance. 

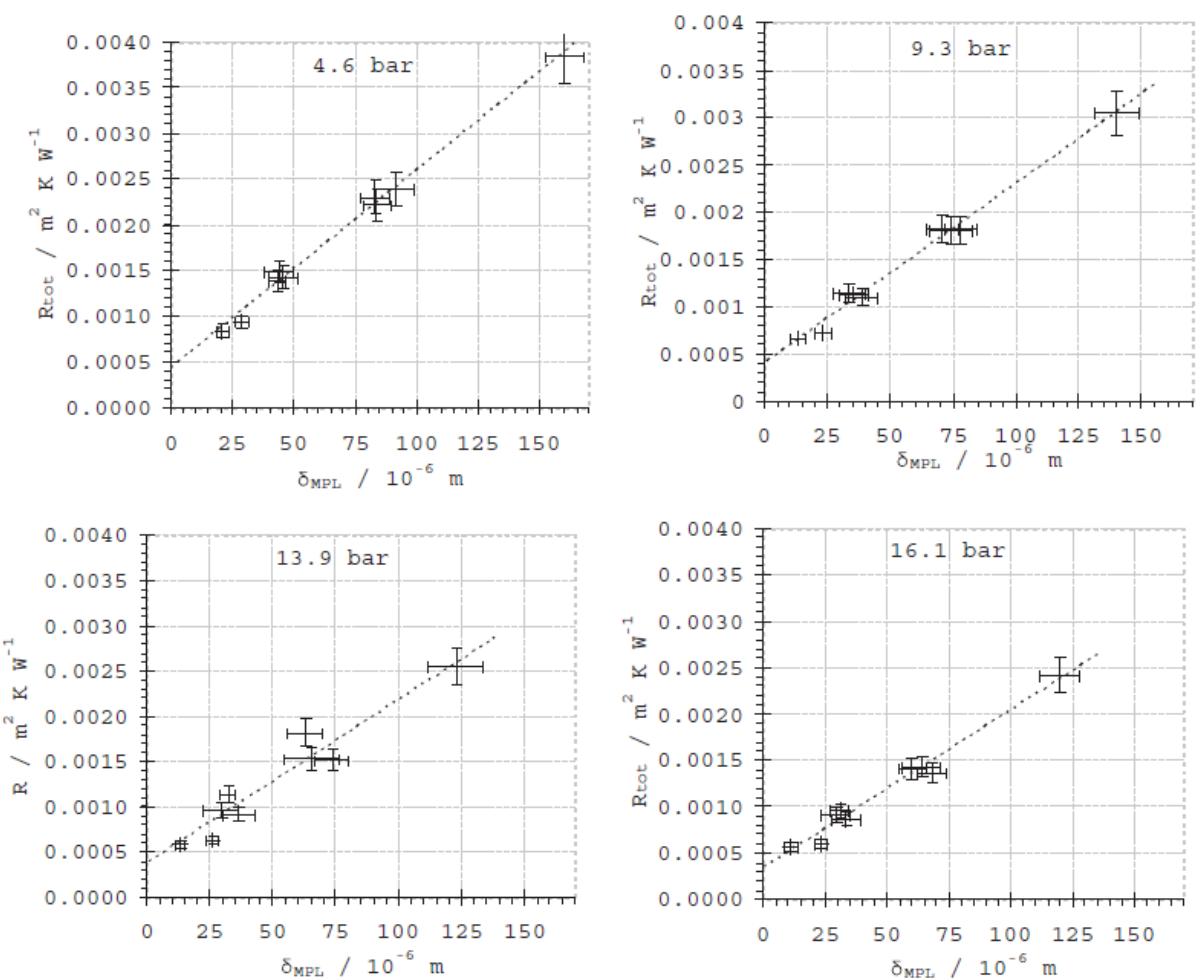

Fig. A.10 - Measured total thermal resistance for single and stacked pairs of the MPL1, MPL2, MPL2 with a layer of aluminium on top of each.
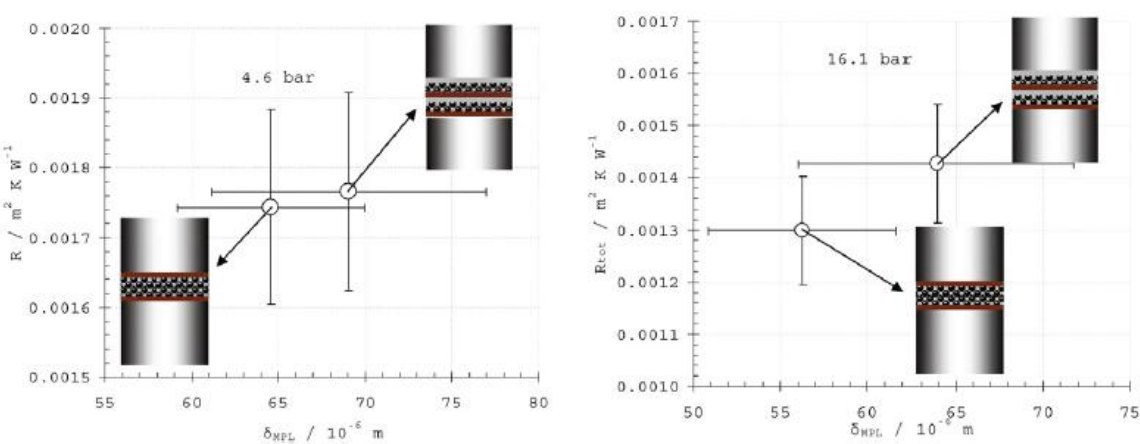

Fig. A.11 - The measured total resistance of the standard stacked pair and the pair facing each other at 4.6 and 16.1 bar compaction pressure.

Another method to check the importance of the stack internal contact resistance is to measure the total thermal resistance of a pair when stacked as described in the standard procedure and when omitting any of the aluminium films and allowing the two MPL to face each other. In several previous studies it is shown that the thermal contact resistance between either two PTL [31] or between two MPL [12] are negligible. If the thin aluminium film in the stack has a negligible resistance contribution (in itself or by contacts to the MPL etc.) removing should in principle give the same measurements of the total thermal resistance of a pair. When doing this validation exercise for the lower pressure, MPL that were compressed at 16.1 bar was used, meaning that after having done the full cycle of measurements we remeasured the standard stack at low pressure and then re-remeasured the thermal 
resistance of this pair when removing the aluminium films and having the MPL facing each other. The result for the lower (4.6 bar) and the higher (16.1 bar) pressure are shown in Fig. A.11. Regardless of the compaction pressure, the measured total thermal resistance is the same for both the configurations. Thus a second validation of the chosen procedure is given. 


\section{References}

[1] Kjelstrup S, Røsgjorde A. Local and total entropy production and heat and water fluxes in a one-dimensional polymer electrolyte fuel cell. $J$ Phys Rev B 2005;109:9020.

[2] Burheim O, Vie P, Møller-Holst S, Pharoah J, Kjelstrup S. A calorimetric analysis of a polymer electrolyte fuel cell and the production of $\mathrm{H}_{2} \mathrm{O}_{2}$ at the cathode. Electrochim Acta 2010;5:935e42.

[3] Thomas A, Maranzana G, Didierjean S, Dillet J, Lottin O. Thermal effect on water transport in proton exchange membrane fuel cell. Fuel Cells 2012;12:212e24.

[4] Ramousse J, Lottin O, Didierjean S, Maillet D. Heat sources in proton exchange membrane (PEM) fuel cells. J Power Sources 2009;192:435e41.

[5] Burheim O, Kjelstrup S, Pharoah J, Vie P, Møller-Holst S. Calculation of reversible electrode heats in the proton exchange membrane fuel cell from calorimetric measurements. Electrochim Acta 2011;5:935e42.

[6] Thomas A, Maranzana G, Didierjean S, Dillet J, Lottin O. Measurements of electrode temperatures, heat and water fluxes in PEMFCs: conclusions about transfer mechanisms. J Electrochem Soc 2013;160(2):F191e204.

[7] Kjelstrup S, Vie PJS, Akyalcin L, Zefaniya P, Pharoah J, Burheim OS. The seebeck coefficient and the peltier effect in a polymer electrolyte membrane cell with two hydrogen electrodes. Electrochim Acta 2013;99:166e75.

[8] Kinoshita K. Electrochemical oxygen technology. New York: Interscience; 1992.

[9] El-kharouf A, Mason TJ, Brett DJ, Pollet BG. Ex-situ characterisation of gas diffusion layers for proton exchange membrane fuel cells. J Power Sources 2012;218:393e404.

[10] El-Kharouf A, Chandan A, Hattenberger M, Pollet B. Proton exchange membrane fuel cell degradation and testing: review. $J$ Energy Inst 2012;85(4):188e200.

[11] Zamel N, Li X. Effective transport properties for polymer electrolyte membrane fuel cells e with a focus on the gas diffusion layer. Prog Energy Combust Sci 2013;39:146.

[12] Burheim OS, Ellila G, Fairweather JD, Labouriau A, Kjelstrup S, Pharoah JG. Ageing and thermal conductivity of porous transport layers used for PEM fuel cells. J Power Sources 2013;221:356e65.

[13] Unsworth G, Zamel N, Li X. Through-plane thermal conductivity of the microporous layer in a polymer electrolyte membrane fuel cell. Int $J$ Hydrogen Energy 2012;37(6):5161e9.

[14] Cindrella L, Kannan A, Lin J, Saminathan K, Ho Y, Lin C, et al. Gas diffusion layer for proton exchange membrane fuel cells e a review. J Power Sources 2009;194(1):146e60.

[15] Wang X, Zhang H, Zhang J, Xu H, Tian Z, Chen J, et al. Microporous layer with composite carbon black for PEM fuel cells. Electrochim Acta 2006;51(23):4909e15. [16] Ji Y, Luo G, Wang C-Y. Pore-level liquid water transport through composite diffusion media of PEMFC. J Electrochem Soc 2010;157:B1753. 
[17] Park S, Lee J-W, Popov BN. Effect of carbon loading in microporous layer on PEM fuel cell performance. J Power Sources 2006;163:357e63.

[18] Park S, Lee J-W, Popov BN. Effect of PTFE content in microporous layer on water management in PEM fuel cells. J Power Sources 2008;177:457e63.

[19] Kim T, Lee S, Park H. A study of water transport as a function of the microporous layer arrangement in PEMFCs. Int $J$ Hydrogen Energy 2010;35:8631e43.

[20] Wu R, Zhu X, Liao Q, Wang H, dong Ding Y, Li J, et al. A pore network study on the role of micro-porous layer in control of liquid water distribution in gas diffusion layer. Int J Hydrogen Energy 2010;35:7588e93.

[21] Weng F-B, Hsu C-Y, Su M-C. Experimental study of micro-porous layers for PEMFC with gradient hydrophobicity under various humidity conditions. Int $\mathrm{J}$ Hydrogen Energy 2011;36:13708e14.

[22] Nitta I, Himanen O, Mikkola M. Thermal conductivity and contact resistance of compressed gas diffusion layer of PEM fuel cell. Fuel Cells 2008;8:111e9.

[23] He G, Yamazaki Y, Abudula A. A three-dimensional analysis of the effect of anisotropic gas diffusion layer (GDL) thermal conductivity on the heat transfer and two-phase behavior in a proton exchange membrane fuel cell (PEMFC). J Power Sources 2010;195:1551e6o.

[24] Pharoah J, Karan K, Sun W. On effective transport coefficients in PEM fuel cell electrodes: Anisotropy of the porous transport layer. $J$ Power Sources 2006;161:214e24.

[25] Danes F, Bardon J. Thermal conductivity of the carbon felts, strongly anisotropic insulants: modelling of heat conduction by solid phase. Uses of carbon felts for high temperature insulation are reviewed. Eff Raw Mater Fabr 2008;36:200e8.

[26] Sadeghi E, Bahrami M, Djilali N. Analytic determination of the effective thermal conductivity of PEM fuel cell gas diffusion layers. J Power Sources 2008;179:200e8.

[27] Sadeghi E, Djilali N, Bahrami M. A novel approach to determine the in-plane thermal conductivity of gas diffusion layers in proton exchange membrane fuel cells. J Power Sources 2011;196:3565-71.

[28] Teertstra P, Karimi G, Li X. Measurement of in-plane effective thermal conductivity in PEM fuel cell diffusion media. Electrochim Acta 2011;56:1670-5.

[29] Zamel N, Litovsky E, Shakhshir S, Li X, Kleiman J. Measurement of in-plane thermal conductivity of carbon paper diffusion media in the temperature range of $20{ }^{\circ} \mathrm{C}$ to $\mathrm{b} 120$ O C. Appl Energy 2011;88:3042־50.

[30] Ramousse J, Lottin O, Didierjean S, Maillet D. Estimation of the thermal conductivity of carbon felts used as PEMFC gas diffusion layers. Int $J$ Thermal Sci 2008;47:1e6.

[31] Burheim O, Vie P, Pharoah J, Kjelstrup S. Ex-situ measurements of throughplane thermal conductivities in a polymer electrolyte fuel cell. J Power Sources 2010;195:249e56. 
[32] Burheim O, Lampert H, Pharoah J, Vie P, Kjelstrup S. Throughplane thermal conductivity of PEMFC porous transport layers. Fuel Cell Sci Technol 2011;8:1e11. Art. no. 021013 .

[33] Wang Y, Gundevia M. Measurement of thermal conductivity and heat pipe effect in hydrophilic and hydrophobic carbon papers. Int $\mathrm{J}$ Heat Mass Transf 2013;60:134e42.

[34] Khadelwal M, Mench MM. Direct measurement of throughplane thermal conductivity and contact resistance in fuel cell materials. J Power Sources 2006;161:1106e15.

[35] Yableckia J, Nabovati AA, Bazylak A. Modeling the effective thermal conductivity of an anisotropic gas diffusion layer in a polymer electrolyte membrane fuel cell. J Electrochem Soc 2012;159:B647e53.

[36] Vie P, Kjelstrup S. Thermal conductivities from temperature profiles in the polymer electrolyte fuel cell. Electrochim Acta 2004;49:1069e77.

[37] Ihonen J, Mikkola M, Lindhberg G. The flooding of gas diffusion backing in PEFCs; physical and electrochemical characterisation. $J$ Electrochem Soc 2004;151:A1152e61.

[38] Zamel N, Litovsky E, Shakhshir S, Li X, Kleiman J. Measurement of the through-plane thermal conductivity of carbon paper diffusion media for the temperature range from -50 to b120 Oc. Int J Hydrogen Energy 2011;36:12618e25.

[39] Su H-N, Liao S-J, Wu Y-N. Significant improvement in cathode performance for proton exchange membrane fuel cell by a novel double catalyst layer design. J Power Sources 2010;195:3477e80.

[40] Bapat C, Thynell S. Anisotropic heat conductions effects in proton-exchange membrane fuel cells. ASME J Heat Transfer 2007;129:1109e18.

[41] Zhang G, Kandlikar SG. A critical review of cooling techniques in proton exchange membrane fuel cell stacks. Int $J$ Hydrogen Energy 2012;37:2412e29.

[42] Springer TE, Zawodzinski TA, Gottesfeld SS. Polymer electrolyte fuel cell model. J Electrochem Soc 1991;138:2334e43.

[43] Reucroft P, Rivin D, Schneider N. Thermodynamics of nafion-vapor interactions. I. Water vapor. Polymer 2002;43:5157e61.

[44] West RC. CRC e handbook of physical and chemical properties. 58th ed. CRC Press, Inc; 1977-1978.

[45] Sadeghi E, Djilali N, Bahrami M. Effective thermal conductivity and thermal contact resistance of gas diffusion layers in proton exchange membrane fuel cells. Part 2: hysteresis effect under cyclic compressive load. J Power Sources 2010;195:8104e9.

[46] Turhan A, Heller K, Brenizer J, Mench M. Passive control of liquid water storage and distribution in a PEFC through flow-field design. J Power Sources 2008;180:773e83. 